\title{
Comparative Analysis of Quality of Service for Various Service Classes in WiMAX Network using NS-3
}

\author{
Gaurav Sharma \\ CSE and IT Department \\ BBSB Engineering College \\ Fatehgarh Sahib, India
}

\author{
Rishma Chawla \\ CSE and IT Department \\ RIET College \\ Phagwara, India
}

\begin{abstract}
Broadband access is an important requirement to satisfy user demands and support a new set of real time services and applications. WiMAX, as a Broadband Wireless Access solution for Wireless Metropolitan Area Networks, covering large distances with high throughput and is a promising technology for Next Generation Networks. Nevertheless, for the successful deployment of WiMAX based solutions, Quality of Service (QoS) is a mandatory feature that must be supported. Quality of Service (QoS) is an important consideration for supporting variety of applications that utilize the network resources. These applications include voice over IP, multimedia services, like, video streaming, video conferencing etc. In this paper the performances of the MPEG-4 High quality video traffic over a WiMAX network using various service classes has been investigated. To analyze the QoS parameters, the WiMAX module developed based on popular network simulator NS-3 is used. Various parameters that determine QoS of real life usage scenarios and traffic flows of applications is analyzed. The objective is to compare different types of service classes with respect to the QoS parameters, such as, throughput, packet loss, average delay and average jitter.
\end{abstract}

Keywords: BE, ertPS, nrtPS, QoS, rtPS, UGS, WiMAX, NS-3

\section{INTRODUCTION}

WiMAX [8], Worldwide Interoperability for Microwave Access are designed to deliver a metro area broadband wireless access (BWA) service. It is based on Institute of Electrical and Electronics Engineers (IEEE) 802.16 standard. The technology provides basic Internet Protocol (IP) connectivity to the user. The rapid growth of new services based on multimedia applications such as Voice over IP, Audio and Video Streaming, Video Conferencing, File Transfer, e-mail etc. has created a demand for last mile broadband access. The various advantages of BWA include rapid deployment, high scalability, and lower maintenance and upgrade costs, and granular investment to match market growth. Various multimedia applications along with the common email, file transfer and web browsing applications are becoming increasingly popular. These applications send large audio and video streams with variable bandwidth and delay requirements.

On the other hand, remote monitoring of critical services, electronic commerce and banking applications, as well as, network control and signaling do not need strict bandwidth guarantees due to the burst nature of the data transfer. These applications also require reliable and prompt packet routing. The presence of different kinds of applications in a network, results in heterogeneous traffic load. The traffic from different applications may require certain type of quality of service. IEEE 802.16/WiMAX provides different service flow classes for different applications to enhance the performance. In this paper, the Quality of Service (QoS) asprescribed in the WiMAX networks is studied and performance analysis of different service flows is compared with respect to QoS parameters like throughput, packet loss, average delay and average jitter.

\section{WiMAX NETWORK IP-BASED ARCHITECTURE}

The WiMAX End-to-End Network Systems Architecture document [2] defines the WiMAX Network
Reference Model (NRM). It is a logical representation of the network architecture. The NRM identifies functional entities and reference points over which interoperability is achieved. The architecture has been developed with the objective of providing unified support of functionality needed in a range of network deployment models and usage scenarios.

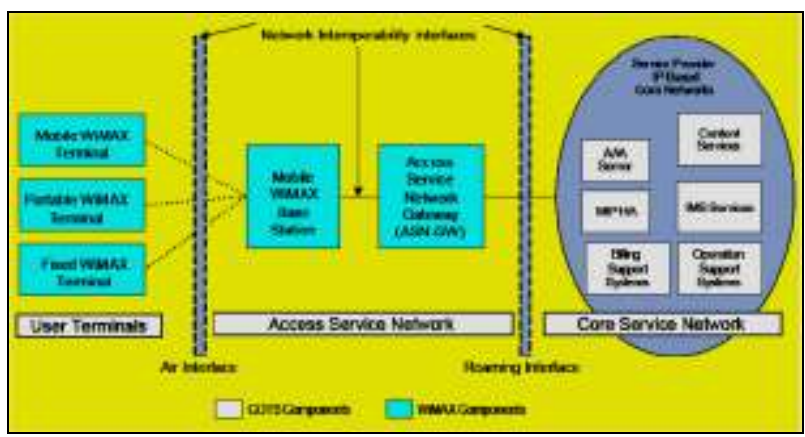

Figure 1: WiMAX Network IP-Based Architecture [3]

Figure 1 shows basic components of a WiMAX network. The subscriber stations (SS) are connected over the air interface to the base station (BS). The base station is part of the Access Service Network (ASN) and connects to the Connectivity Service Network (CSN) through the ASN Gateway. In generic telecommunication terminology, ASN is equivalent to RAN (Radio Access Network) and CSN is equivalent to Core.

The ASN performs the following main functions:

i. WiMAX Layer 2 and Layer 3 connectivity with the subscriber stations, including IP address allocation.

ii. Network discovery and selection of an appropriate network service provider that the subscriber station accesses. 
iii. Radio Resource Management

The CSN performs the following main functions:

i. Provides internet access

ii. Authentication, Authorization and Accounting

iii. Policy and admission control based on user subscription profiles

\section{QUALITY OF SER VICE IN IEEE 802.16/WiMAX NETWORK}

In 1994, QoS, in the field of telephony, was defined in the International Telecommunication Union (ITU) Recommendation E.800. This definition is very broad, listing 6 primary components: Support, Operability, Accessibility, Retainability, Integrity and Security. In 1998, the ITU published a document discussing QoS in the field of data networking. The term Quality of Service refers to the probability of the telecommunication network meeting a given traffic contract. In the field of packet-switched networks and computer networking it is used informally to refer to the probability of a packet succeeding in passing between two points in the network. Although the name suggests that it is a qualitative measure of how reliable and consistent a network is, there are a number of parameters that can be used to measure it quantitatively. These include throughput, transmission delay or packet delay, delay jitter, percentage of packets lost etc. QoS mechanism is added in the layer 2 i.e. Media Access Control (MAC) layer architecture of IEEE 802.16 standards. MAC layer is responsible for scheduling of bandwidth for different users. The MAC layer performs bandwidth allocation based on user requirements as well as their QoS profiles. The standard is designed to support a wide range of applications. These applications may require different levels of QoS. To accommodate these applications, the 802.16 standard has defined five service flow classes where each service flow is characterized by a mandatory set of QoS parameters, which is tailored to best describe the guarantees required by the applications that the service flow class is designed for. Furthermore, for uplink connections, it also specifies which mechanisms to use in order to request bandwidth. This enables end-to-end IP based QoS. The service flows can be created, changed, or deleted by the issuing Dynamic Service Addition (DSA), Dynamic Service Change (DSC), and Dynamic Service Deletion (DSD) messages.

Table 1. Five service flows defined in MAC layer architecture of IEEE 802.16 standards

\begin{tabular}{|l|l|l|}
\hline Service Class & Application & Delay Sensitivity \\
\hline UGS & Voice & No Delay \\
\hline $\begin{array}{l}\text { rtPS (real-time } \\
\text { Polling Service) }\end{array}$ & Streaming video & High \\
\hline $\begin{array}{l}\text { ertPS (extended } \\
\text { real- time Polling } \\
\text { Service) }\end{array}$ & $\begin{array}{l}\text { Voice over IP with } \\
\text { silence suppression }\end{array}$ & Very high \\
\hline $\begin{array}{l}\text { nrtPS (non real- } \\
\text { time Polling } \\
\text { Service) }\end{array}$ & $\begin{array}{l}\text { FTP, messaging, } \\
\text { games }\end{array}$ & Moderate \\
\hline Best Effort & $\begin{array}{l}\text { Email, } \\
\text { Browsing }\end{array}$ & Low \\
\hline
\end{tabular}

Each of these actions can be initiated by the Subscriber Station (SS) or the Base Station (BS) and are carried out through a two or three-way-handshake. The five service flows defined in MAC layer architecture of IEEE 802.16 standards are summarized in Table 1 .

\subsection{Unsolicited Grant Services (UGS):}

UGS is designed to support constant bit rate (CBR) services, such as T1/E1 emulation, and voice over IP (VoIP) without silence suppression. It offers transmission authorization on a periodic basis. UGS traffic is scheduled in a way that SS has a dedicated slot (of fixed size) in which it transmits, and never has to ask for bandwidth for this service (except when creating flow). This guarantees the data rate for the connection.

\subsection{Real-Time Polling Services (rtPS)}

rtPS is designed to support real- time services that generate variable size data packets on a periodic basis, such as MPEG video or VoIP with silence suppression. In opposition to UGS, the SS should perform explicit requests, which will simply an increase of the overhead and latency i.e. in rtPS class, BS provides periodic uplink request opportunities that match the requested real-time needs in which a SS can specify the desired bandwidth.

\subsection{Extended Real Time Polling Service (ertPS)}

Another service type called ertPS (Extended rtPS) was introduced to support variable rate real-time services such as VoIP and video streaming. It has an advantage over UGS and rtPS for VolP applications because it carries lower overhead than UGS and rtPS.

\subsection{Non-Real-Time Polling Services (nrtPS)}

The nrtPS is designed to support non-real-time services that require variable size data grant burst types on a regular basis. It is very similar to rtPS but SSs can ask for bandwidth in a random fashion. In the nrtPS class, BS polls on a regular basis (minimum traffic rate is achieved, but not latency).

\subsection{Best Effort (BE) Services}

BE services are typically provided by the Internet today for Web surfing. By definition this is a class of service that does not provide any guarantees in terms of throughput and / or delays. For BE, SS may use contention request opportunities, as well as unicast polls when the BS sends them. Since BS doesn't need to poll for BE traffic, a long period may pass before BE packets are sent, especially when network is congested

\section{RELATED WORK}

Talwalkar and Iliyas [3] have analyzed quality of service in WiMAX networks. In their analysis, a WiMAX module is developed based on popular network simulator NS2. In their simulation they have used VoIP and video traffic for the analysis of QoS for three service classes (BE, UGS, rtPS) in WiMAX networks, while the QoS for nrtPS and ertPS has not been analyzed because the WiMAX module developed does not support the nrtPS and ertPS service classes.

In 2010 QoS deployment over a cellular WiMAX network [4] was examined. The author has compared the performance obtained using two different QoS configurations differing from the delivery service class used to transport VoIP traffic, i.e. UGS or ertPS. OPNET modeller version 14.5 
with WiMAX module capability was been used for the simulation.

The analysis of various critical QoS parameters like throughput, average jitter and average delay for VoIP using NOAH as protocol in NS-2 simulator has also been done [5]. Their simulation focuses on the QoS parameters for BE service class.

H. Abid, H. Raja, A. Munir, J. Amjad, A. Mazhar and D. Lee [6] performed a performance analysis when multimedia contents are transferred over WiMAX network using $\mathrm{BE}$ and ertPS service classes. The analysis of QoS service for WiMAX network using MATLAB for simulation of WiMAX network and AODV as the routing protocol has also been done. This time the performance analysis focuses on UGS service class only [7].

Anouari and Haqiq [8] in 2012 investigated the performances of the most common VoIP codecs, which are G.711, G.723.1 and G.729 over a WiMAX network using various service classes and NOAH as a transport protocol. To analyze the QoS parameters, the popular network simulator NS-2 was used. Various parameters that determine QoS of real life usage scenarios and traffic flows of applications is analyzed. Their objective was to compare different types of service classes with respect to the QoS parameters, such as, throughput, average jitter and average delay. The service classes for which the QoS was analyzed includes BE, UGS and rtPS, again nrtPS and ertPS service classes are not included in the work.

Table 2 Comparison of various techniques, services classes and simulation tools used

\begin{tabular}{|l|l|l|}
\hline \multicolumn{1}{|c|}{ Technique Service } & Simulator \\
\hline $\begin{array}{l}\text { Performance analysis of } \\
\text { Video Conferencing and } \\
\text { Multimedia application } \\
\text { Services over WiMAX [11] }\end{array}$ & $\begin{array}{l}\text { BE, UGS, } \\
\text { rtPS NS-2 }\end{array}$ & \\
\hline $\begin{array}{l}\text { Measuring data and voip } \\
\text { traffic in wimax networks [4] }\end{array}$ & UGS, rtPS & OPNET \\
\hline $\begin{array}{l}\text { Analysis of VoIP traffic in } \\
\text { WiMAX using NS2 simulator } \\
\text { [5] }\end{array}$ & BE & \\
\hline $\begin{array}{l}\text { Performance Analysis of } \\
\text { WiMAX Best Effort and } \\
\text { erPS Service Classes for } \\
\text { Video Transmission [6] }\end{array}$ & & NE, ertPS \\
\hline $\begin{array}{l}\text { Performance Analysis of QoS } \\
\text { Parameters for Wimax } \\
\text { Networks [7] UGS }\end{array}$ & \\
\hline $\begin{array}{l}\text { Performance Analysis of } \\
\text { VoIP Traffic in WiMAX } \\
\text { using Various Service Classes } \\
\text { [8] }\end{array}$ & $\begin{array}{l}\text { BE, UGS and } \\
\text { rtPS NS-2 }\end{array}$ \\
\hline
\end{tabular}

From the Table 2 it is concluded that the performance analysis for service classes like BE, UGS, rtPS have been carried out by different authors using different simulating tools like NS-2, MATLAB, OPNET. This gave us the idea to analyze the performance analysis of nrtPS service class for WiMAX network and analyze the comparative results for the same.

This work focuses on comparative performance analysis of different service classes which includes BE, rtPS, nrtPS and UGS when video traffic is transferred over the WiMAX network. To analyze the QoS parameter, simulation based on popular network simulator NS-3 is used. Various parameters that determine QoS of real life usage scenarios and traffic flows of applications are analyzed. The goal is to compare different types of service flows with respect to QoS parameters, such as, throughput, packet loss, average delay and average jitter.

\section{SIMULATION DETAILS}

To analyze QoS in a network it is necessary to study real life scenarios. The simulation set up would reflect the actual deployment of the WiMAX network. Based on the network reference model described earlier Figure 2 shows the setup that will be used. There are multiple SS's in the range of a base station. The base station is connected to the core network. The focus of analysis will be the point-to-multipoint connection between the subscriber stations and the base station. Various types of traffic can be set up from one or many subscriber station(s) and a base station to mimic real life scenarios. Video streaming will be used to provide real-time variable bit rate traffic i.e. watching videos online.

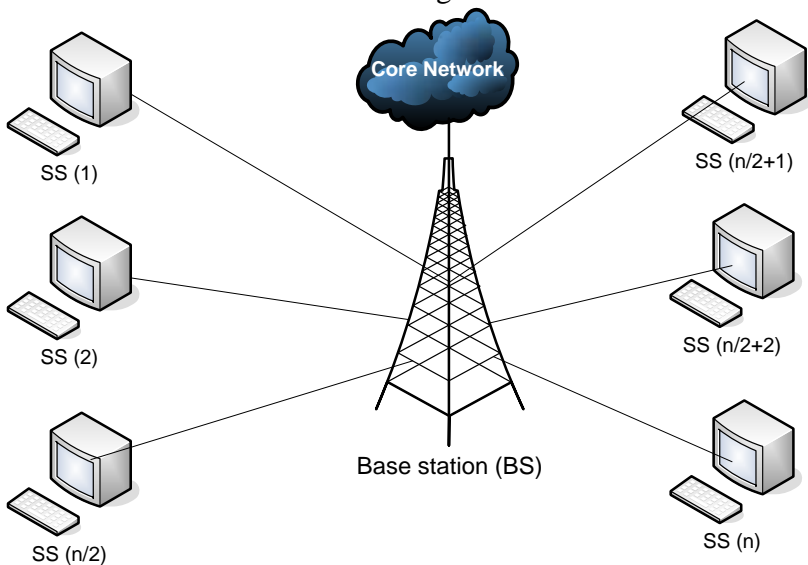

Figure 2. Simulation Setup

NS-3 is a discrete event simulator, that can execute $\mathrm{C}++$ and Python based scripts. NS-3 is likely to be the most popular tool for simulation purposes because of being open source, having well managed source code and easy to use languages like $\mathrm{C}++$. It provides support for UDP and MAC protocols over wired and wireless networks.

Amine Ismail [9] worked on the WiMAX module for NS-3, that suits the requirements of this thesis work and with the help of the basic WiMAX scripts available in the module it became possible to write the scripts. Most network elements in NS-3 simulator are developed as classes, in object-oriented fashion. It is freely distributed and all the source code is available.

Figure 3 shows the basic structure of NS-3 architecture and setup used in this thesis work. The network topology and traffic scenarios etc. are specified in the C++ script. The $\mathrm{C}++$ library has all the implementation details. When NS-3 is run, the resulting simulation data can be obtained in a text file format trace. This file contains time stamp and information about each packet that is sent, received or dropped. It also has information about the packet size, type of packet etc. A base station and subscriber station can be set up as a node in NS-3. As the number of nodes in the simulation increase, the packets that are sent and received increases. This makes the trace file very large. To aid in extracting the right data out from the file, Php script is written. This extracted data is used to prepare charts and graphs for the results of the simulation. 


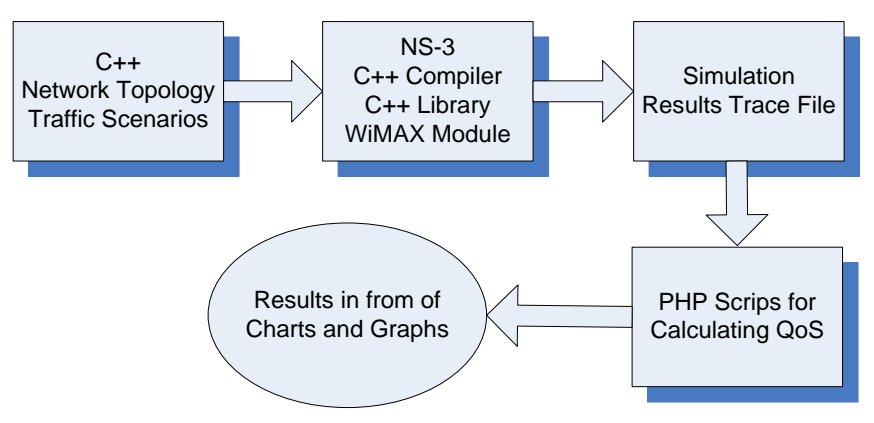

Figure 3 Ns-3 Architecture and set up

The NS-3 WiMAX module has the distinction between nodes. While setting up the node, it could be classified as a base station as opposed to a subscriber node.

The WiMAX module developed by Amine Ismail is used, that work on IEEE 802.16e standard. The WiMAX module simulates both physical (PHY) and MAC layers based on IEEE 802.16e standard.

After setting up NS-3 and compiling the WiMAX module it was discovered that there are several memory leaks in the simulation. For a single node the simulation would run properly but as the nodes increased, so would the packet traffic. This would eventually cause the simulation to stop to run as it would run out of dynamic memory necessary to allocate new packets. Several memory leaks issues were fixed during the course of experimentation, to make the simulation traffic.

As mentioned earlier, simulation scenario for NS-3 is defined by a C++ script. In a typical model, the nodes in the network are setup, whose properties are changed to base station and subscriber stations. The subscriber stations are associated with the base station. Application traffic agent(s) are created and are attached to the source node(s). An example of a traffic Agent could be a VBR traffic agent. On the top of the traffic agent, an application which generates required traffic is created. Half of the subscriber stations will act as the traffic sources and another half of the subscriber stations will act as traffic sinks. For that purpose only an even number of subscriber stations like, 2, 4, 6, 8 and 10 are considered. The focus of the study will be the measurement and analysis of the QoS parameters using the information gathered from the total downlink packets, from base station to subscriber station, and uplink packets, from subscriber station to base station.

Sink Nodes

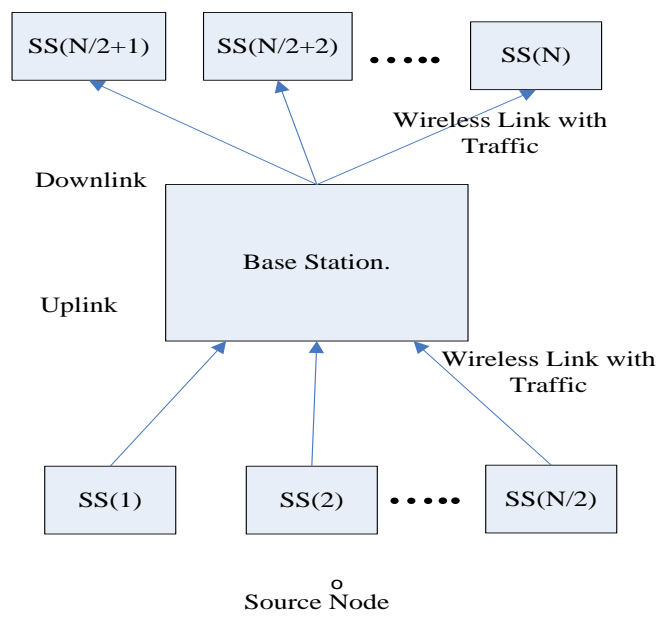

Figure 4 NS-3 Simulation Example
Figure 4 displays a typical example of the NS-3 setup. In the WiMAX module, the simple nodes are created, that are converted into Base Station (BS) nodes and Subscriber Station (SS) nodes using the WiMAX module that creates Subscriber Stations as DEVICE_TYPE_SUBSCRIBER_STATION and the base station is created as DEVICE_TYPE_BASE_STATION. Thereafter, the SS and BS nodes are converted into network devices and IP addresses are assigned to them. This is done to provide the network facilities to the SS \& BS nodes. Mobility can also be applied to the nodes or they can be kept static. After that half of the SS nodes are converted into UDP servers and reset half of the SS nodes are converted into UDP clients. The UDP trace file (video streaming traffic) is applied on the UDP server(s) that is transmitted by the UDP servers and received by UDP client(s),for the duration for which the UDP application runs. The service flows can be changed as per the requirement. The types of service flows that are supported by the WiMAX module are BE, rtPS, nrtPS and UGS. At this point, ertPS service flow is not supported.

The C++ script was parameterized. The input parameters that were varied, for example, number of nodes in the simulation, service flow classes were passed in as a parameter while running the simulation for easy execution.

\section{QUALITY OF SERVICE PARAMETERS}

QoS provisioning encompasses providing Quality of Service to the end user in terms of several generic parameters. The perceived quality of service can be quantitatively measured in terms of several parameters. In the analysis, the throughput, average delay, average jitter and packet loss were considered.

\subsection{Throughput}

Throughput is the amount of number of packets effectively transferred in a network, in other words throughput is data transfer rate that are delivered to all terminals in a network. It is measured in terms of packets per second or per time slot It is a measure of the date rate (bits per second) generated by the application. Equation 1 shows the calculation for throughput TP, where PacketSize $_{i}$ is the packet size of the ith packet reaching the destination, PacketStart ${ }_{o}$ is the time when the first packet left the source and PacketArrival $_{n}$ is the time when the last packet arrived.

$$
T P=\frac{\sum_{i} \text { PacketSize }_{i}}{\text { PacketArrival }_{n}-\text { PacketStart }_{0}}
$$

Equation 1: Throughput Calculation

From the trace file, based on the packet ID, each data packet was kept track of. The time a packet is sent, the time when the packet was received and the packet size was stored for all packets that reached the destination. To calculate throughput, the size of each packet was added. This gave the total data that was transferred. The total time was calculated as the difference between the time the first packet started and the time the last packet reached the destination. Thus throughput is equal to the total data transferred divided by the total time it took for the transfer. 


\subsection{Average Delay or latency}

Delay or latency would be time taken by the packets to transverse from the source to the destination. The main sources of delay can be further categorized into sourceprocessing delay, propagation delay, network delay and destination processing delay. Equation 2 show the calculation for Average Delay, where PacketArrival is the time when packet "i" reaches the destination and PacketStart ${ }_{i}$ is the time when packet " $i$ " leaves the source. " $n$ " is the total number of packets.

$$
\text { AverageDelay }=\frac{\sum_{i} \text { PacketArrival }_{i}-{\text { Packet } \text { Start }_{i}}_{\text {Par }}}{n}
$$

Equation 2: Average Delay

From the trace file, difference between the start time of the packet and time when the packet reaches destination is calculated. The average of all these times gives the average delay.

\subsection{Jitter or Delay Variation}

Jitter can be observed as the end-to-end delay variation between two consecutive packets. The value of jitter is calculated from the end to end delay. Jitter reveals the variations in latency in the network caused by congestion, route changes, queuing, etc. Delay variation is the variation in the delay introduced by the components along the communication path. It is the variation in the time between packets arriving. Jitter is commonly used as an indicator of consistency and stability of a network. Measuring jitter is critical element to determining the performance of network and the QoS the network offers. Equation 3 shows the steps for calculation of average jitter. It is the average of the absolute difference in the time it took for successive packets to reach the destination.

AverageJitter $=\frac{\sum_{i} \mid \begin{array}{l}\left(\text { PacketArrival }_{i+1}-\text { PacketStart }_{i+1}\right)- \\ \left(\text { PacketArrival }_{i}-\text { PacketStart }_{i}\right)\end{array}}{n-1}$

Equation 3: Average Jitter

There are five packets that arrive at the destination with slightly varying delays. Note that the numbers mentioned here do not represent any of the actual data. They are thought about to make the calculations clear. In the last column, the absolute value of difference in successive packets is calculated. Thus average jitter is the average of delay difference in successive packets.

The packets should arrive at the same delay. The delay entries in "Delay" column will be identical. This will have the delay variation to be zero, implying no jitter.

\subsection{Packet loss or corruption rate}

Packet loss affects the perceived quality of the application. Several causes of packet loss or corruption would be bit errors in an erroneous wireless network or insufficient buffers due to network congestion when the channel becomes overloaded. Equation 4 shows the simple equation to calculate packet loss. It is the sum of all the packets that do not reach the destination over the sum of the packets that leave the destination.

$$
\text { PacketLoss }=\frac{\sum \text { LostPacketSize }_{i}}{\sum_{\text {Equation 4: Packet Loss }} \text { PacketSize }_{j}} \times 100
$$

Packet loss calculation is relatively simple. The sum of the packet size of all packets that are sent is calculated. Next, the sum of all packets that are received is calculated. The difference in the two values gives the data that was lost. The ratio of total data lost and the total data that was sent gives the packet loss.

A php script was written to analyze the ns-3 trace files and calculate the four parameters.

\section{SIMULATION RESULTS}

To analyze the quality of service in WiMAX network, real life use case is considered. WiMAX provides basic IP connectivity. With the availability of a larger data pipe, viewing videos over the internet is common application these days. So video streaming is analyzed. In this section, the simulation results for QoS parameters, obtained for video traffic, are presented.

A number of subscriber stations generating video traffic are set up in this experiment. The performance of QoS service flows in terms of the QoS parameters was analyzed. Video streaming is Variable Bit Rate (VBR) traffic. Unlike Constant Bit Rate (CBR) the packet size varies based on each frame type e.g. in case of a MPEG traffic, the 'I' and ' $\mathrm{B}$ ' frames are smaller in size than a ' $P$ ' frame. Similarly for H.263 traffic, there are 'I' frames, 'P' frames and 'PB' frames. For the analysis using the simulation, a high quality MPEG-4 data stream was used. MPEG-4 is designed as a simple and straight forward video coding with enhanced compression performance and to provide a network friendly video representation. MPEG-4 video streaming is need to be packetized for transportation over networks. The transport protocol for MPEG-4 is Real-time Transport Protocol (RTP). The VBR frame stream for the high quality MPEG-4 encoded Mr. Bean movie was obtained from the following website in [10]. Using this information, a trace file is generated. This trace file is attached to the UDP agent as a traffic source. The trace file is in binary format and contains information of the time, frame number, frame type and packet size. Analysis is done using BE, nrtPS, rtPS and UGS service flows for the video traffic. The parameters analyzed are throughput, packet loss, average jitter and average delay. These parameters are observed for each service flow as the number of nodes with the video traffic increases.

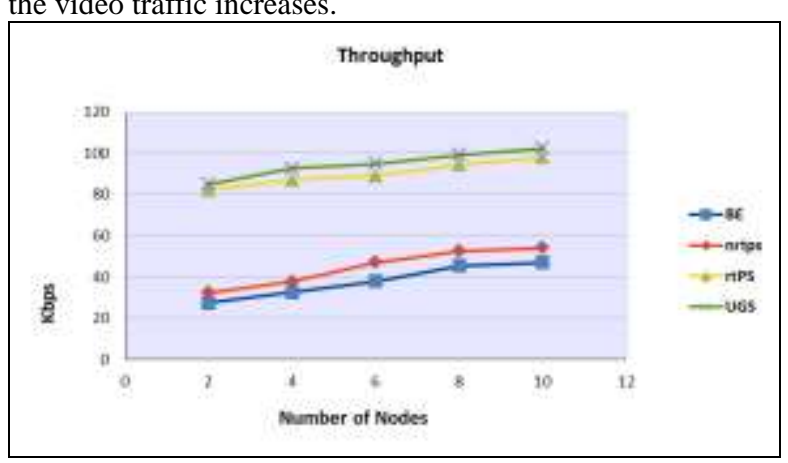

Figure 5: Overall throughput for video traffic as the number of nodes increases

Figure 5 shows the variation of overall throughput for video traffic as the number of nodes increases. The overall throughput of rtPS and UGS service flow is higher than BE and nrtPS service flow. For 10 nodes the value of throughput for BE service flow is lowest. If the throughput using UGS 
service flow is compared with three other service flows i.e. BE, nrtps and rtPS, the UGS service flow provides much higher throughput than BE and nrtPS service flow and nearly equal throughput to the rtPS service flow for the same number of nodes.

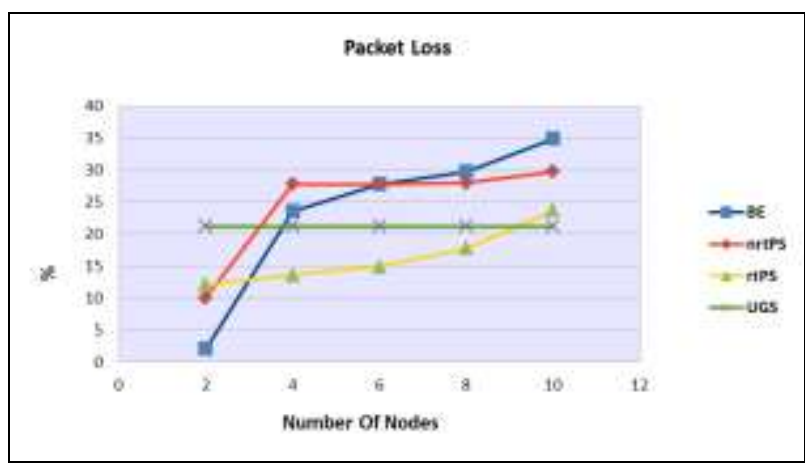

Figure 6 Variation of packet loss for video traffic with all four service flows.

Figure 6 shows the variation in packet loss for video traffic with all four service flows. UGS service flow provides constant packet loss. In case of BE and nrtPS service flow the packet loss increases gradually as the number of nodes streaming video traffic in network increases. The value of packet loss for 2 nodes is much less than the value for 10 nodes for both the service flow i.e. BE and nrtPS. However in case of rtPS service flow there is very slight variation in packet loss as the number of nodes increases from 2 to 10 and the value of packet loss is lowest than all three service flows for increasing number of nodes.

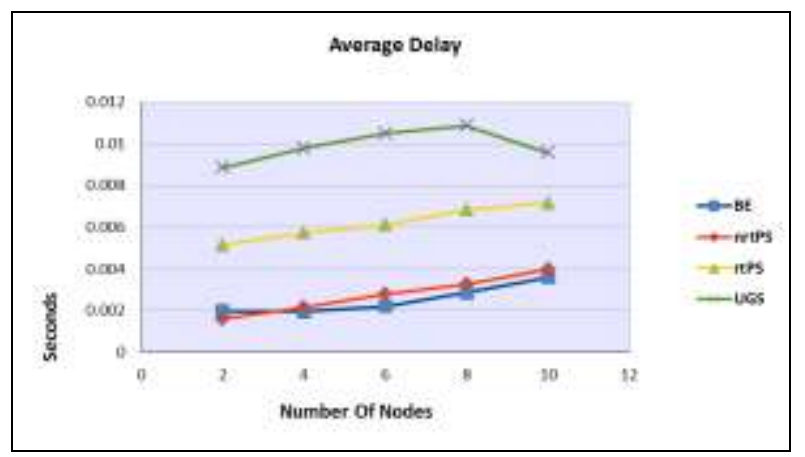

Figure 7 Average delay for four service flows

Average Figure 7 shows the average delay for the four service flows i.e. BE, nrtPS, rtPS and UGS as the number of nodes increase. UGS has higher delay as compared with BE, nrtPS and rtPS flows, although the delay variation is less for UGS. This is because of UGS offers fixed grants on a periodic basis. There is no bandwidth request mechanism in BE traffic. Data is sent whenever resources are available and not required by any other scheduling-service classes. As the number of nodes increase the average delay increases rapidly for $\mathrm{BE}$ service flow as compared to rtPS service flow. The value of average delay is higher in rtPS service flow as compared to nrtPS service flow but in case of rtPS the variation in delay value is comparatively much less than the nrtPS sevice flow. Similar to BE service flow the value of delay increases rapidly in nrtPS service flow as the number of nodes streaming video traffic increases.

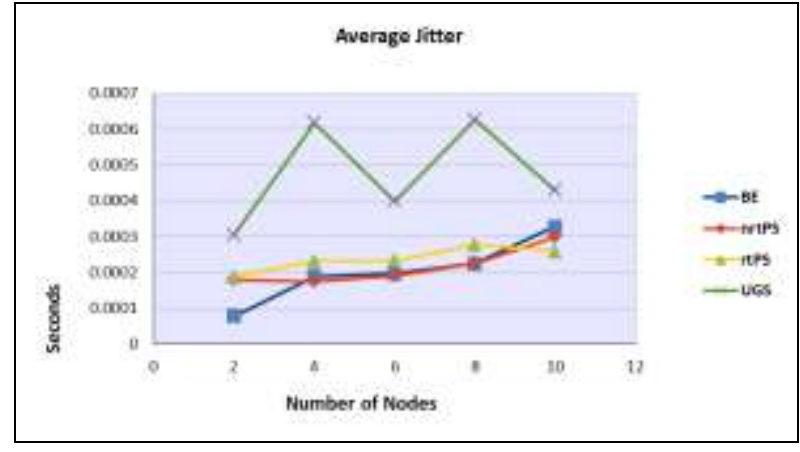

Figure 8 Variation in average jitter with all four service flows

Figure 8 shows the variation in average jitter with all four service flows. From the Figure 8 it can be seen that the value of jitter does not show any relation with number of nodes for UGS flow. However, rtPS service flow has lowest jitter value than all the four service flows i.e. BE, nrtPS and UGS for maximum number of nodes i.e. 10 nodes and it remains more or less constant. For small number of nodes, $\mathrm{BE}$ service flow has the lowest jitter. As the number of nodes increases, the jitter value starts increasing rapidly. Thus when lot of network resources are available video traffic over BE service flow introduces the least jitter. However, as the nodes increase, the network resources get divided between all the nodes. Hence, it results into the increased jitter. However in case of rtPS service flows the average jitter is having a small variation with the increase in the number of nodes and is almost constant.

\section{CONCLUSION}

The performance analysis of different service flows namely BE, nrtps, rtPS and UGS, on QoS parameters like throughput, packet loss, average delay and average jitter was analyzed and compared when video traffic is passed with increasing number of nodes over WiMAX network. During the analysis rtPS service flow comes out to be better than all other three service flow for average jitter and packet loss. The variation in value of average jitter is very less in case of rtPS service flow and it has least jitter value for maximum number of nodes i.e. 10 than all other service flows. In case of average delay the value for rtPS service flow is high than the BE and nrtPS service flow but there is very slight variation in value with increasing number of nodes which increases rapidly in case of other two. The delay is maximum in UGS service flow for video traffic as it is a VBR traffic. rtPS service flows shows the least packet loss while streaming video traffic with increased number of nodes and throughput is much higher as compared to BE and nrtPS service flow and is nearly equal to UGS service flow with increased number of nodes. As the UGS service flow does not utilize the network resources effectively when the traffic is not Constant Bit Rate (CBR) traffic and streaming video traffic is Variable Bit Rate (VBR) traffic. The bandwidth can be periodically requested in rtPS service flow instead of fixed bandwidth already being allocated, which may or may not get used. Thus, by considering all the results, it can be concluded that for streaming video traffic rtPS service flow is best suited.

\section{FUTURE SCOPE}

Video streaming was considered in the current analysis. Further analysis could be done for other applications including VOIP, video telephony which combines video traffic and VOIP traffic, File Transfer Protocol (FTP) traffic etc. The 
WiMAX module used in the analysis did not support ertPS service flow that is defined by the IEEE 802.16 standards. ertPS service flow is designed for applications which generate variable rate traffic which are delay dependent. An example of such traffic is VOIP with silence suppression.

\section{REFERENCES}

[1]. Retnasothie, F. E., Ozdemir, M. K., Cek, T.Y., Celebi, H., Zhang, J., and Muththaiah, R., "Wireless IPTV over WiMAX: Challenges and Applications",Proceedings of IEEE WAMICON, [2006].

[2]. WiMAX End-to-End Network Systems Architecture Stage 2: Architecture Tenets, Reference Model and Reference Points," WiMAX Forum, December, [2005]

[3]. Talwalkar, R. A. and Ilyas, M.,"Analysis of Quality of Service (QoS) in WiMAX Networks", IEEE International Conference on Networking, [2008].

[4]. Adhicandra, I., "Measuring data and voip traffic in wimax networks," Arxiv Preprint arXiv:1004.4583, [2010].

[5]. Joshi, D. and Jangale, S., "Analysis of VoIP traffic in WiMAX using NS2 simulator" International Journal of Advanced Research in Computer Science and Electronics Engineering, Vol. 1, Issue 2, April [2012].

[6]. Abid, H., Raja, H., Munir, A., Amjad, J., Mazhar, A. and Lee, D., "Performance Analysis of WiMAX Best Effort and ertPS Service Classes for Video Transmission", ICCSA, Issue 3, Page(s): 368-375, [2012].

[7]. Vikram, M. and Gupta, N.,"Performance Analysis of QoS Parameters for Wimax Networks." International Journal of Engineering and Innovative Technology (IJEIT) Volume 1, Page(s): 105-110, [2012].

[8]. Anouari, T. and Haqiq, A., "Performance Analysis of VoIP Traffic in WiMAX using Various Service Classes", International Journal of Computer Applications (0975 8887), Volume 52, No. 20, August [2012].

[9]. Ismail, M. A., Piro, G., Grieco, L. A. and Turletti, T. “ An Improved IEEE 802.16 WiMAX Module for the NS3 Simulator", International Conference on Simulation Tools and Techniques (SIMUTools'10), March, [2010].

[10]. "MPEG-4 and H.263 Video Traces for Network Performance Evaluation" (Master's thesis) Athamneh K. http://trace.eas.asu.edu/TRACE/pics/FrameTrace/mp4/V erbose_bean.dat

[11]. Kaarthick, Yeshwenth, V. J., Nagarajan. N. and Rajeev, "Performance analysis of Video Conferencing and Multimedia application Services over WiMAX", IEEE International Advance Computing Conference, Page(s):1109-1123, March [2009]. 